KODAI MATH. SEM. REP.

20 (1968), 94-102

\title{
ON HYPERSURFACES WITH CONSTANT $k$-TH MEAN CURVATURE
}

\author{
By MARIKO TANI
}

\section{$\S 1$. Introduction.}

The study arising from the formula of Minkowski has been pursued by Liebmann [5], Süss [6], Hsiung [1], [2], Katsurada [3], [4], Yano [7], [8] and others. Most of their works have been combined with the condition that the first mean curvature is constant. The purpose of the present paper is to get the integral formulas for a hypersurface whose $k$-th mean curvature ${ }^{1)}$ is constant and also to show the integral formula given by Hsiung [2] and Katsurada [3] in a different way.

\section{§. Notations and general formulas on hypersurfaces.}

Let $M^{n+1}$ be an $(n+1)$-dimensional orientable Riemannian manifold with local coordinates $\left\{x^{\lambda}\right\}{ }^{2}{ }^{2} \quad$ Let $g_{\lambda \mu}, \nabla_{\lambda}, K_{\lambda \mu \nu}{ }^{\omega}$, and $K_{\mu \nu}$ be a Riemannian metric, the operator of covariant differentiation with respect to the Riemannian metric, curvature tensor and Ricci tensor, respectively.

We consider a closed orientable hypersurface $V^{n}$ in a Riemannian space $M^{n+1}$ whose local parametric expression is

$$
x^{\lambda}=x^{\lambda}\left(u^{i}\right)
$$

where $u^{\left.i{ }^{3}\right)}$ are local coordinates in $V^{n}$. If we put

$$
B_{i}{ }^{2}=\frac{\partial x^{\lambda}}{\partial u^{2}}
$$

then $B_{i}{ }^{\lambda}$ are $n$ linearly independent vectors tangent to $V^{n}$. The first fundamental tensor $g_{j i}$ of $V^{n}$ is given by

$$
g_{j i}=g_{\lambda \mu} B_{j}{ }^{\lambda} B_{i}{ }^{\mu} .
$$

Since $M^{n+1}$ is oriented, there is a uniquely determined unit normal vector $C^{\lambda}$ for each point of $V^{n}$, and we have

Received July 17, 1967.

1) $k$-th mean curvature will be defined in $\S 4$.

2) Here and in the sequel Greek indices $\lambda, \mu, \nu, \cdots$ run over the range $1, \cdots, n, n+1$.

3) Here and henceforth Latın indices $i, j, k, \cdots$ run over the range $1, \cdots, n$. 


$$
g^{j i} B_{\jmath}{ }^{\lambda} B_{i}{ }^{\mu}=g^{\lambda \mu}-C^{\lambda} C^{\mu} \text {. }
$$

Denoting by $\nabla_{3}$ the operation of van der Waerden-Bortolotti covariant differentiation along the hypersurface $V^{n}$, we have the equations of Gauss and Weingarten;

$$
\begin{aligned}
\nabla_{j} B_{i}{ }^{2} & =h_{j i} C^{\lambda}, \\
\nabla_{j} C^{\lambda} & =-h_{j}{ }^{k} B_{k}{ }^{2}
\end{aligned}
$$

where $h_{j i}$ are components of the second fundamental tensor of $V^{n}$ and $h_{j}{ }^{k}=h_{j i} g^{i k}$.

We also have the equations of Gauss and Codazzi:

$$
K_{\lambda \mu \nu \omega} B_{k}{ }^{\lambda} B_{\jmath}{ }^{\mu} B_{i}{ }^{\nu} B_{h}{ }^{\omega}=K_{k j i h}-\left(h_{k h} h_{j i}-h_{j h} h_{k i}\right),
$$

$$
K_{\lambda \mu \nu \omega} B_{k}{ }^{\lambda} B_{j}{ }^{\mu} B_{i}{ }^{\nu} C^{\omega}=\nabla_{k} h_{j i}-\nabla_{j} h_{k \imath},
$$

where $K_{k j i n}$ is the curvature tensor of the hypersurface $V^{n}$. Transvecting this equation with $g^{j i}$ we get

$$
K_{\mu \nu} B_{k}{ }^{\mu} C^{\nu}=\nabla_{k} h_{j}{ }^{j}-\nabla_{j} h_{k}{ }^{j} .
$$

The principal curvatures $a_{(1)}, \cdots, a_{(n)}$ of the hypersurface $V^{n}$ are the roots of the determinant equation

$$
\left|h_{j i}-a g_{j i}\right|=0
$$

\section{§ 3. Algebraic preliminaries.}

Let $a_{(1)}, \cdots, a_{(n)}$ be the $n$ principal curvatures at the point $\mathrm{P}$ of $V^{n}$ and consider the $k$-th elementary symmetric function of $a_{(1)}, \cdots, a_{(n)}$

$$
S_{k}=\sum_{i_{1}<\cdots<i_{k}} a_{\left(i_{1}\right)} \cdots a_{\left(i_{k}\right)}, \quad s_{0}=1 .
$$

If we put

$$
P_{(k)}=\sum_{i=1}^{n}\left(a_{(i)}\right)^{k}
$$

then we have the relations between $S_{k}$ and $P_{(k)}$, so-called Newton formula, that is

$$
\begin{aligned}
& P_{(1)}-S_{1}=0, \\
& P_{(2)}-S_{1} P_{(1)}+2 S_{2}=0, \\
& P_{(3)}-S_{1} P_{(2)}+S_{2} P_{(1)}-3 S_{3}=0, \\
& \cdots \cdots \cdots \cdots \cdots \cdots \cdots \cdots \cdots \cdots \cdots \cdots \cdots \cdots \cdots \cdots \cdots \cdots \cdots \cdots \cdots \cdots \cdots \cdots \cdots \cdots \\
& P_{(n)}-S_{1} P_{(n-1)}+S_{2} P_{(n-2)}-\cdots+(-1)^{n-1} S_{n-1} P_{(1)}+n(-1)^{n} S_{(n)}=0 .
\end{aligned}
$$

Therefore we can express $S_{k}$ in the terms of $P_{(1)}, \cdots, P_{(k)}$ as follows: 


$$
\begin{aligned}
& S_{1}=P_{(1)}, \\
& S_{2}=\frac{1}{2}\left(-P_{(2)}+P_{(1)}^{2}\right), \\
& S_{3}=\frac{1}{3 !}\left(2 P_{(3)}-3 P_{(1)} P_{(2)}+P_{(1)}^{3}\right), \\
& S_{4}=\frac{1}{4 !}\left(-6 P_{(4)}+8 P_{(3)} P_{(1)}-6 P_{(1)}^{2} P_{(2)}+3 P_{(2)}{ }^{2}+P_{(1)}^{4}\right) \text {, } \\
& S_{k}=\sum_{t_{1}+2 t_{2}+\cdots+n t_{n}=k} \frac{(-1)^{t_{1}+\cdots+t_{n}+k}}{\left(t_{1} !\right)\left(t_{2} !\right) \cdots\left(t_{n} !\right) 2^{t_{2} \cdots n^{t_{n}}}} P_{(1)}{ }^{t_{1} \cdots P_{(n)}}{ }^{t_{n}}, \\
& S_{n}=\sum_{t_{1}+2 t_{2}+\ldots+\cdots+n t_{n}=n} \frac{(-1)^{t_{1}+\cdots+t_{n}+n}}{\left(t_{1} !\right)\left(t_{2} !\right) \cdots\left(t_{n} !\right) 2^{t_{2} \cdots n^{t_{n}}}} P_{(1)}{ }^{t_{1} \cdots P_{(n)}{ }^{t_{n}}}
\end{aligned}
$$

On the other hand we have the following identity

$$
\frac{n-k}{n} S_{1} S_{k}-(k+1) S_{k+1}=\frac{1}{n} \sum_{\substack{i_{1}<i_{2} \\ \imath_{3}<\cdots<i_{k+1}}}\left(a_{\left(i_{1}\right)}-a_{\left(i_{2}\right)}\right)^{2} a_{\left(i_{3}\right)} \cdots a_{\left(i_{k+1}\right)}
$$

which will be used in $\S 5$.

\section{§4. Formulas in $M^{n+1}$ admitting a conformal vector field.}

In this section we assume that the Riemannian manifold $M^{n+1}$ admits a conformal vector field $v^{2}$, i.e. $v^{2}$ is a vector field which satisfies

$$
\nabla_{\mu} v_{\lambda}+\nabla_{\lambda} v_{\mu}=2 \rho g_{\mu \lambda}
$$

where $v_{\mu}=g_{\mu \lambda} v^{\lambda}$ and $\rho$ is $(1 /(n+1)) V_{\lambda} v^{\lambda}$. Putting

$$
v^{\lambda}=B_{i}^{\lambda} v^{i}+\alpha C^{\lambda}
$$

and transvecting (4.1) with $B_{j}{ }^{\mu} B_{i}{ }^{\lambda}$, we obtain

$$
\nabla_{j} v_{i}+\nabla_{i} v_{\jmath}=2 \rho g_{j i}+2 \alpha h_{j i}
$$

from which

$$
\nabla_{s} v^{s}=n \rho+\alpha P_{(1)}
$$

If we apply Green's formula to this formula, we have

$$
\int\left(n \rho+\alpha P_{(1)}\right) d V=0
$$


For simplicity we fix the notations as follows.

$$
\begin{aligned}
& H_{(m)}{ }^{{ }^{1} \imath_{m+1}}=h^{\imath_{112}} h_{i_{2}}{ }^{{ }^{3} \cdots} h_{i_{m}}{ }^{{ }^{2 m+1}}, \quad H_{(0) i}{ }^{j}=h_{i}{ }^{3}, \\
& v_{(m)}{ }^{{ }^{m+1}}=h_{i_{1}}{ }^{{ }^{2}} h_{i_{2}}{ }^{23} \cdots h_{i_{m}}{ }^{2 m+1} v^{{ }^{21}}, \quad v_{(0)}{ }^{\imath}=v^{\imath}, \\
& K_{\imath j k 0}=K_{\lambda \mu \nu \omega} B_{i}{ }^{\lambda} B_{\jmath}{ }^{\mu} B_{k}{ }^{\nu} C^{\omega} \text {, } \\
& K_{0 \imath j 0}=K_{\lambda \mu \nu \omega} C^{\lambda} B_{i}{ }^{\mu} B_{\jmath}{ }^{\nu} C^{\omega} \text {, } \\
& K_{i 0}=K_{\mu \nu} B_{i}{ }^{\mu} C^{\nu}, \\
& K_{00}=K_{\mu \nu} C^{\mu} C^{\nu} \text {. }
\end{aligned}
$$

Let us consider all of the following formulas for any integer $k(0 \leqq k \leqq n)$

$$
\begin{aligned}
& \int \nabla_{s} v_{(k)}{ }^{s} d V=0, \\
& \int \nabla_{s} P_{(1)} v_{(k-1)}^{s} d V=0, \\
& \int \nabla_{s} P_{(1)}{ }^{t_{1}} \ldots P_{(n)}{ }^{t_{n}} v_{(k-2)}{ }^{s} d V=0 \quad\left(t_{1}+\cdots+n t_{n}=2\right),
\end{aligned}
$$

(4. 5) $)_{k}$

$$
\begin{aligned}
& \int V_{s} P_{(1)}{ }^{t_{1}} \cdots P_{(n)}{ }^{t_{n}} v_{(k-m)}{ }^{s} d V=0 \quad\left(t_{1}+\cdots+n t_{n}=m\right),
\end{aligned}
$$

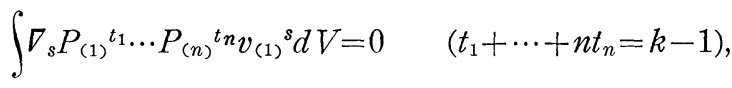

$$
\begin{aligned}
& \int \nabla_{s} P_{(1)}{ }^{t_{1}} \cdots P_{(n)}{ }^{t_{n}} v_{(0)}{ }^{s} d V=0 \quad\left(t_{1}+\cdots+n t_{n}=k\right) .
\end{aligned}
$$

Forming the following expression of the integrands in $(4.5)_{k}$,

$$
A_{k}=\sum_{\substack{t_{1}+2 t_{2}+\cdots+m=k \\ 0 \leq m \leq k \\ t_{i} \geqq 0}} b_{(k)}\left(t_{1}, \cdots, t_{n}\right) \nabla_{s} P_{(1)}{ }^{t_{1}} \cdots P_{(n)}{ }^{t_{n}} v_{(m)} s
$$

where

$$
b_{(k)}\left(t_{1}, \cdots, t_{n}\right)=\frac{(-1)^{t_{1}+\cdots+t_{n}+k}}{\left(t_{1} !\right) \cdots\left(t_{n} !\right) 2^{t_{2} \cdots} n^{t_{n}}}
$$

we find that $A_{k}(0 \leqq k \leqq n)$ does not contain the terms of the derivative of $P_{(j)}{ }^{t}$; that is the terms of the form $\left(\nabla_{s} P_{(1)}{ }^{t_{1}} \ldots P_{(n)}{ }^{t_{n}}\right) v_{(m)}{ }^{s}$ are cancelled each other for all $j, t$ and $m$. In order to check up this fact we note the following equalities. 


$$
\begin{aligned}
& \nabla_{s} P_{(1)}{ }^{t_{1}} \cdots P_{(l)}{ }^{t_{l}} \cdots P_{(n)}{ }^{t_{n}} \\
& =\sum_{l=1}^{n} t_{l} P_{(1)}{ }^{t_{1}} \ldots P_{(l-1)}{ }^{t_{l-1}} P_{(l)}{ }^{t_{l}-1}\left(\nabla_{s} P_{(l)}\right) P_{(l+1)}{ }^{t_{l+1}} \ldots P_{(n)}{ }^{t_{n}}, \\
& \nabla_{s} v_{(m)}{ }^{s}=\left(\nabla_{s} h_{i_{1}}{ }^{22} \cdots h_{i_{m}}{ }^{s}\right) v^{{ }_{11}}+\left({h_{i_{1}}}^{{ }^{2} \cdots} h_{i_{m}}{ }^{s}\right) \nabla_{s} v^{{ }^{11}} \\
& =\sum_{l=1}^{m} H_{(l-1) \imath_{1}}{ }^{\imath}\left(\nabla_{s} H_{\imath_{l}}{ }^{\left.{ }^{2} l+1\right)} H_{(m-l) \imath_{l+1}}{ }^{s} v^{\imath_{1}}+H_{(m) \imath_{1}}{ }^{s}\left(\rho \delta_{s}{ }^{{ }^{1} 1}+\alpha h_{s}{ }^{{ }^{1}}\right)\right. \\
& =\sum_{l=1}^{m} H_{(l-1) \imath_{1}}{ }^{\imath} l\left(\nabla_{\imath_{l}} h_{s}{ }^{\imath l+1}-K_{\left.\imath_{l} s^{{ }{ }^{l+1+1}}\right)}\right) H_{(m-l) \imath_{l+1}}{ }^{s} v^{\imath_{1}}+\rho P_{(m)}+\alpha P_{(m+1)} \\
& =\sum_{l} \frac{1}{m-l+1}\left(\nabla_{\imath l} P_{(m-l+1)}\right) v_{(l-1)} \imath^{\imath}
\end{aligned}
$$

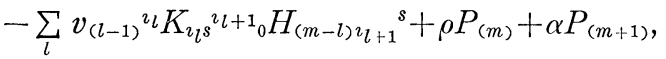

$$
\begin{aligned}
& \nabla_{s} v_{(1)}{ }^{s}=\left(\nabla_{s} h_{i}{ }^{s}\right) v^{2}+h_{i}{ }^{s}\left(\nabla_{s} v^{i}\right) \\
& =\left(\nabla_{i} h_{s}{ }^{s}-K_{i 0}\right) v^{2}+h_{i}{ }^{s}\left(\rho \delta_{s}{ }^{2}+\alpha h_{s}{ }^{i}\right) \\
& =\left(\nabla_{i} P_{(1)}\right) v^{2}-K_{i 0} v^{2}+\rho P_{(1)}+\alpha P_{(2)} \text {. }
\end{aligned}
$$

These equations are derived from (2.1), (2.2), (4.2) and (4.4).

Moreover the coefficients $b_{(k)}\left(t_{1} \cdots t_{n}\right)$ introduced above are nothing but the numbers in the formula (3.1). Therefore we get

$$
\begin{aligned}
A_{k}= & S_{k}\left(n \rho+\alpha P_{(1)}\right)-S_{k-1}\left(\rho P_{(1)}+\alpha P_{(2)}\right)+\cdots+(-1)^{k}\left(\rho P_{(k-1)}+\alpha P_{(k)}\right) \\
& +S_{k-1} K_{i 0} v_{i}-S_{k-2}\left(K_{l i j 0} H_{(0)}{ }^{j i} v^{l}+K_{i 0} v_{(1)}{ }^{i}\right) \\
& +S_{k-3}\left(K_{l i j 0} H_{(1)}{ }^{j i} v^{l}+K_{l i j 0} H_{(0)}{ }^{j i} v_{(1)}{ }^{l}+K_{i 0} v_{(2)}{ }^{i}\right) \\
& -\cdots+(-1)^{k+1}\left(K_{l i j 0} H_{(k-1)}^{j i} v^{l}+K_{l i j 0} H_{(k-2)}^{j i} v_{(1)}{ }^{l}+\cdots\right. \\
& \left.+K_{l i j 0} H_{(1)}{ }^{j i} v_{(k-2)}{ }^{l}+K_{i 0} v_{(k-1)}{ }^{i}\right) \\
= & (n-k) \rho S_{k}+\alpha(k+1) S_{k+1}+S_{k-1} K_{i 0} v^{\imath} \\
& +\sum_{l=2}^{k}(-1)^{l+1} S_{k-l}\left(\sum_{m=0}^{l-2} H_{(l-m-1)}^{j i} v_{(m)}^{h} K_{h \imath j 0}+v_{(l-1)}{ }^{i} K_{i 0}\right) .
\end{aligned}
$$

By virtue of Green's integral formula, we have

$$
\begin{aligned}
& \int\left\{(n-k) \rho S_{k}+\alpha(k+1) S_{k+1}+S_{k-1} K_{i 0} v^{\imath}\right. \\
& \left.\quad+\sum_{l=2}^{k}(-1)^{l+1} S_{k-l}\left(\sum_{m=0}^{l-2} H_{(l-m-1)}{ }^{j i} v_{(m)}{ }^{h} K_{h \imath j 0}+v_{(l-1)}{ }^{2} K_{i 0}\right)\right\} d V=0 .
\end{aligned}
$$


If we assume that $S_{k}$ is constant for any fixed $k$, then from (4.3), (4.6), (4.7) and (3. 2), we obtain

$$
\begin{aligned}
& \int\left\{\frac{\alpha}{n} \sum_{\substack{{ }_{3}<\cdots<i_{2} \\
<\cdots-1}}\left(a_{\left(i_{1}\right)}-a_{\left(i_{2}\right)}\right)^{2} a_{\left(i_{3}\right)} \cdots a_{\left(i_{k+1}\right)}-S_{k-1} K_{i 0} v^{\imath}\right. \\
& \left.\quad+\sum_{l=2}^{k}(-1)^{l} S_{k-l}\left(\sum_{m=0}^{l-2} H_{(l-m-1)}{ }^{\imath \jmath} v_{(m)}{ }^{h} K_{h \imath j 0}+K_{i 0} v_{(l-1)}{ }^{2}\right)\right\} d V=0 .
\end{aligned}
$$

The $k$-th mean curvature $M_{k}$ of the hypersurface is defined by

$$
\left(\begin{array}{l}
n \\
k
\end{array}\right) M_{k}=S_{k}
$$

where $\left(\begin{array}{l}n \\ k\end{array}\right)$ denotes the binomial coefficient. Then we have

$$
M_{1} M_{k}-M_{k+1}=\frac{k !(n-k-1) !}{n n !} \sum_{\substack{i_{1}<i_{2} \\{ }_{3}<\cdots<i_{k+1}}}\left(a_{\left(i_{1}\right)}-a_{\left(i_{2}\right)}\right)^{2} a_{\left(i_{3}\right)} \cdots a_{\left(i_{k+1}\right)} .
$$

For a space of constant curvature, (4.7) becomes

$$
\int\left(\rho M_{k}+\alpha M_{k+1}\right) d V=0 .
$$

and from (4.8) we get

THEOREM 1. Let $M^{n+1}$ be an orientable Riemannian manifold of constant curvature which admits a conformal vector field $v$ and $V^{n}$ be a closed orientable hypersurface in $M^{n+1}$. Suppose that there exists an integer $k$ such that

1) $M_{k}$ is constant,

2) $M_{1} M_{k}-M_{k+1} \geqq 0$

and that the inner product of normal vector $C^{\lambda}$ and $v^{\lambda}$ has definite sign.

Then $V^{n}$ is totally umbilic.

The result for a space of constant curvature are given in [2] and [3].

\section{$\S 5$. Concircular scalar field.}

In this last section we get some results on the hypersurface in a Riemannian manifold $M^{n+1}$ which admits a special conformal vector field. That is, we investigate the case in which $M^{n+1}$ admits a non constant scalar field $v$ such that

$$
\nabla_{\mu} \nabla_{\lambda} v=f(v) g_{\mu \lambda}
$$

where $f(v)$ is a differentiable function of $v$. We call such scalar field $v$ a concircular field. If we put

$$
v_{\lambda}=\nabla_{\lambda} v, \quad g^{\mu} v_{\mu}=v^{\lambda}
$$


we see that $v^{\lambda}$ is a conformal vector.

Putting

$$
v^{2}=B_{i}^{\lambda} v^{2}+\alpha C^{\lambda}
$$

and transvecting (5.1) with $B_{j}{ }^{\mu} B_{i}{ }^{2}$, we have

$$
\nabla_{j} v_{i}=f(v) g_{j i}+\alpha h_{j i}
$$

from which we get

$$
\nabla_{s} v^{s}=n f(v)+\alpha P_{(1)} .
$$

By virtue of Ricci identity we get

$$
K_{\lambda \mu \nu \omega} v^{\omega}=-f^{\prime}(v)\left(v_{\lambda} g_{\mu \nu}-v_{\mu} g_{\lambda \nu}\right),
$$

from which

$$
K_{\mu \nu} v^{\mu}=-n f^{\prime}(v) v_{\nu}
$$

and

$$
K_{\mu \nu} v^{\mu} C^{\nu}=-n \alpha f^{\prime}(v) .
$$

Thus we get

$$
\begin{aligned}
K_{h \imath j 0} v^{h} & =K_{\lambda \mu \nu \omega} v^{h} B_{h^{\lambda}} B_{i}{ }^{\mu} B_{\jmath}{ }^{\nu} C^{\omega} \\
& =-K_{\nu \omega \mu \lambda}\left(v^{\lambda}-\alpha C^{\lambda}\right) B_{i}{ }^{\mu} B_{\jmath}{ }^{\nu} C^{\omega} \\
& =\alpha\left\{f^{\prime}(v) g_{i j}-K_{0 \imath j 0}\right\}
\end{aligned}
$$

and

$$
K_{i 0} v^{\imath}=-\alpha\left(n f^{\prime}(v)+K_{00}\right) .
$$

Then the formula (4.8) can be written as

$$
\begin{aligned}
\int\{\alpha & {\left[\frac{1}{n} \sum_{\substack{\imath_{3}<\cdots<i_{k+1} \\
\imath_{1}<i_{2}}}\left(a_{\imath_{1}}-a_{\imath_{2}}\right)^{2} a_{\imath_{3}} \cdots a_{\imath_{k+1}}+S_{k-1}\left\{(n-k+1) f^{\prime}(v)+K_{00}\right\}\right.} \\
& \left.-\sum_{l=2}^{k}(-1)^{l} S_{k-l} H_{(l-1)}{ }^{\imath j} K_{0 \imath j 0}\right] \\
& \left.-\sum_{l=2}^{k}(-1)^{l} S_{k-l} v_{(l-1)}{ }^{i} K_{i 0}-\sum_{l=3}^{k}(-1)^{l} S_{k-l} \sum_{m=1}^{l-2} v_{(m)}{ }^{h} H_{(l-1-m)}{ }^{j i} K_{h \imath j 0}\right\} d V=0 .
\end{aligned}
$$

From the integral formula (5.2) we have

Theorem 2. Let $M$ be an orientable Riemannian manifold which admits a non constant scalar field $v$ such that 


$$
\nabla_{\mu} \nabla_{\lambda} v=f(v) g_{\mu \lambda}
$$

where $f(v)$ is differentiable function of $v$ and $V^{n}$ be a closed orientable hypersurface in $M$. Suppose that there exists an integer $k$ such that

1) $M_{k}$ is constant,

2) $M_{1} M_{k}-M_{k+1} \geqq 0$,

3)

$$
\begin{gathered}
S_{k-1}\left\{(n-k+1) f^{\prime}(v)+K_{00}\right\}-\sum_{l=2}^{k}(-1)^{l} S_{k-l} H_{(l-1)}{ }^{i j} K_{0 \imath j 0} \geqq 0, \\
\sum_{l=2}^{k}(-1)^{l-1} S_{k-l} v_{(l-1)}{ }^{i} K_{i 0}+\sum_{l=3}^{k}(-1)^{l-1} S_{k-l} \sum_{m=1}^{l-2} v_{(m)}{ }^{h} H_{(l-1-m)}{ }^{j i} K_{h \imath j 0} \geqq 0
\end{gathered}
$$

and that the inner product of normal vector $C^{\lambda}$ and $v^{\lambda}$ has positive sign on $V^{n}$.

Then $V^{n}$ is totally umbilic.

If $M^{n+1}$ admits a special concircular scalar field, then we have

Theorem 3. Let $M^{n+1}$ be an orientable Riemannian manifold which admits a non constant scalar field $v$ such that

$$
\nabla_{\mu} \nabla_{\lambda} v=c v g_{\mu \lambda}, \quad c=\text { const., }
$$

and $V^{n}$ be a closed orientable hypersurface in $M$. Suppose that there exists an integer $k$ such that

1) $M_{k}$ is constant,

2) $M_{1} M_{k}-M_{k+1} \geqq 0$,

3)

$$
\begin{gathered}
S_{k}\left\{(n-k+1) c+K_{00}\right\}-\sum_{l=2}^{k}(-1)^{l} S_{k-l} H_{(l-1)}{ }^{i j} K_{02 j 0} \geqq 0, \\
\sum_{l=2}^{k}(-1)^{l-1} S_{k-l} v_{(l-1)}{ }^{i} K_{i 0}+\sum_{l=3}^{k}(-1)^{l-1} S_{k-l} \sum_{m=1}^{l-2} v_{(m)}{ }^{h} H_{(l-1-m)}{ }^{j i} K_{h i j 0} \geqq 0
\end{gathered}
$$

and that the inner product of normal vector $C^{\lambda}$ and $v^{\lambda}$ has positive sign on $V^{n}$.

Then $V^{n}$ is totally umbilic. If $v$ is not constant on $V^{n}$, then $V^{n}$ is isometric to a sphere.

Yano [8] proved that this theorem is true for $k=1$. In our case, the first half can be deduced from theorem 1 , and then the condition that $S_{k}$ is constant for any $k$, is equivalent to the condition that $S_{1}$ is constant. Therefore theorem 2 is valid for any $k$.

\section{BiBLIOGRAPIIY}

[1] Hsiung, C. C., Some integral formulas for closed hypersurfaces. Math. Scand. 2 (1954), 286-294.

[2] - Some integral formulas for closed hypersurfaces in Riemannian space. Pacific J. Math. 6 (1956), 291-299. 
[3] Katsurada, Y., Generalized Minkowski formulas for closed hypersurfaces in a Riemann space. Annali di Math. 57 (1962), 283-294.

[4] - On a certain property of closed hypersurface in an Einstein space. Comment. Math. Helv. 38 (1964), 165-171.

[5] Liebmann, H., Über die Verbıegung der geschlossenen Flächen posıtıver Krümmung. Math. Ann. 53 (1900), 91-112.

[6] Süss, W., Zur relatıven Differentıalgeometrıe. Tôhoku Math. J. 31 (1929), 202-209.

[7] Yano, K., Closed hypersurfaces with constant mean curvature in a Riemannian manıfold. J. Math. Soc. Japan 17 (1965), 333-340.

[8] Notes on hypersurfaces in a Riemannian manifold. Canadian J. Math. 19 (1967), 439-445.

Department of Mathematics,

Tokyo Institute of Technology. 